\title{
Markov model of data measurement complex for track geometry car
}

\author{
$V$ Pogorelov ${ }^{1, *}$ and $E$ Chub $^{1}$ \\ ${ }^{1}$ Don State Technical University, 1, Gagarin sq., Rostov-on-Don, 344003, Russia
}

\begin{abstract}
A stochastic model of a nonadjustable data measurement complex platform for track geometry cars is introduced. A state vector evaluation algorithm based on the approximation of a posteriori probability density by the system of a posteriori moments is also offered.
\end{abstract}

\section{Introduction}

At present an advanced transportation infrastructure plays a vital part in the general economic progress of any nation. Railroads account for a substantial share of this infrastructure. The transportation industry is continuously struggling with growing cargo and passenger traffic, so the arising issues of time efficiency, safety and reliability cannot be solved without maintaining a high operational quality level of the rolling stock and railroad tracks. In order to ensure safe and reliable railroad functionality a variety of regular safety measures is applied, involving track fitness parameter control and registration activities. Out of all existing track fitness control equipment only track geometry cars (TGC) provide for an overall precision track sub- and superstructure monitoring. The TGC measurement capabilities are based on data measurement complexes (DMC) built upon nonadjustable platforms (NP). These complexes feature a number of apparent advantages. Continually active sensors allow for the recording of some crucial characteristics that otherwise may escape detection by initial orientation systems with adjustable platforms. Current platform orientation data is preserved during the sensor calibration process. There are no additional specific elements of the initial orientation system. Meanwhile the NP TGC performance efficiency is highly dependent on its model's adequacy to the real operational environments. That is why NP TGC movement model designers should remember that the NP own drift rate relates nonlinearly to the accelerations axially directed within the relative gyroscopic coordinate system as well as the fact that the NP sensor measurements bear a stochastic nature. For that matter the objective of this research is to design an efficient NP TGC movement model together with a measurement interference filter algorithm.

\footnotetext{
*Corresponding author: vadim.pogorelov.rnd@gmail.com
} 


\section{NP Movement Equations}

In order to develop an NP movement model the following right-hand coordinate systems (CS) are introduced [1]: $O \xi \eta \zeta$-inertial CS (ICS) $I$; ONLE - Earth-related astronomical CS (ACS) $T$, originating at the platform suspension center; $O X Y Z$ - platform-related gyroscopic CS (GCS) $J, O x_{i} y_{i} z_{i}-i$-gyro unit-related CS. For better NP movement analysis visualization it is assumed that the three corresponding basis vectors of the first three CSs are coincident at the reference time.

Each gyro unit's own drift rate $\omega_{i}$ is correlated by the second degree trimetric polynomial from the axially directed accelerations within the related CS [2]:

$$
\omega=R+U g+g^{T} \hat{\otimes} K g,
$$

where $K$ is a block matrix, $\hat{\otimes}$ is the block multiplication sign,

$$
\begin{gathered}
\omega=\left(\begin{array}{lll}
\omega_{x} & \omega_{y} & \omega_{z}
\end{array}\right)^{T}, g=\left(\begin{array}{lll}
g_{x} & g_{y} & g_{z}
\end{array}\right)^{T}, R=\left(\begin{array}{lll}
R_{x} & R_{y} & R_{z}
\end{array}\right)^{T}, \\
U=\left(\begin{array}{ccc}
-U_{z} & U_{y} & U_{x} \\
-U_{x} & -U_{z} & U_{y} \\
U_{z} & -U_{y} & -U_{x}
\end{array}\right), K=\left(\begin{array}{ccc}
k_{z x} & -k_{z y} & 0 \\
K_{2} \\
-K_{3}
\end{array}\right) K_{1}=\left(\begin{array}{ccc}
0 & 0 & k_{x y} \\
k_{z z}-k_{x x} & 0 & -k_{x z}
\end{array}\right), \\
K_{2}=\left(\begin{array}{ccc}
0 & 0 & -k_{x y} \\
k_{z x} & k_{z y} & k_{z z}-k_{y y} \\
0 & 0 & -k
\end{array}\right), K_{3 z}=\left(\begin{array}{ccc}
-k_{x z} & 0 & k_{x x}-k_{z z} \\
-k_{z y} & 0 & 0 \\
0 & -k_{y x} & -k_{x z}
\end{array}\right),
\end{gathered}
$$

$g_{i}, i=x, y, z$ are the accelerations, directed along the corresponding GCS axes, $R_{i}-$ are the drift rate components unrelated to the accelerations, $U_{i}, k_{i, j}(i, j=x, y, z)-$ are the drift rate polynomial factors, setting up the relationship from accelerations in the first and second degrees accordingly.

The platform drift rate projections may be represented with a system of differential equations in relation to the Euler-Krylov angles $\alpha, \beta, \gamma \quad[3]$ :

$$
\left(\begin{array}{c}
\dot{\alpha} \\
\dot{\beta} \\
\dot{\gamma}
\end{array}\right)=\left(\begin{array}{ccc}
\cos \gamma & -\sin \gamma & 0 \\
\frac{\sin \gamma}{\cos \alpha} & \frac{\cos \gamma}{\cos \alpha} & 0 \\
\sin \gamma \operatorname{tg} \alpha & \cos \gamma \operatorname{tg} \alpha & 1
\end{array}\right)\left(\begin{array}{l}
\omega_{x} \\
\omega_{y} \\
\omega_{z}
\end{array}\right) .
$$

Equations (1), (2) allow to correlate the actual NP orientation, i.e, time variation of EulerKrylov angles $\alpha, \beta, \gamma$, to the current overloads along its axes, but for the moment in parametrical form only. In order to identify the acceleration projections $g_{x}, g_{y}, g_{z}$ on the GCS axis with known acceleration projections $G_{N}, G_{L}, G_{E}$ on the ACS axis a matrix rotations correlation will be employed. Trihedron $T$ of the ACS rotates in relation to trihedron $I$ of the ICS around Axis mundi at the rate $\Omega_{3}$, hence 


$$
T=V I
$$

where

$$
V=\left(\begin{array}{ccc}
\left(1-\cos \Omega_{3} t\right) \cos ^{2} \varphi+\cos \Omega_{3} t & \left(1-\cos \Omega_{3} t\right) \frac{\sin 2 \varphi}{2} & -\sin \varphi \sin \Omega_{3} t \\
\left(1-\cos \Omega_{3} t\right) \frac{\sin 2 \varphi}{2} & \left(1-\cos \Omega_{3} t\right) \sin ^{2} \varphi+\cos \Omega_{3} t & \cos \varphi \sin \Omega_{3} t \\
\sin \varphi \sin \Omega_{3} t & -\cos \varphi \sin \Omega_{3} t & \cos \Omega_{3} t
\end{array}\right)-\quad \text { is the }
$$

finite rotation matrix for the ACS trihedron [2,3], $t-$ is rotation time, $\varphi$ is the known latitude of the object's mass center.

The rotation of the platform-related trihedron $J$ of the GCS, in relation to trihedron $I$ may be described in its turn with the help of the first degree rotation matrix $D$ :

$$
J=D I
$$

where

$$
D=\left(\begin{array}{ccc}
\cos \beta \cos \gamma & \sin \alpha \sin \beta \cos \gamma+\cos \alpha \sin \gamma & -\cos \alpha \sin \beta \cos \gamma+\sin \alpha \sin \gamma \\
-\cos \beta \sin \gamma & -\sin \alpha \sin \beta \sin \gamma+\cos \alpha \cos \gamma & \cos \alpha \sin \beta \sin \gamma+\sin \alpha \cos \gamma \\
\sin \beta & -\sin \alpha \cos \beta & \cos \alpha \cos \gamma
\end{array}\right) .
$$

Consequently, the rotation of the platform trihedron $J$ in relation to the Earth trihedron $T$ may be represented by the following matrix equality:

$$
J=D V^{T} T
$$

where $V^{T}$ is the transposed matrix $V$.

It follows from (4) and (5), that the acceleration projections $g_{x}, g_{y}, g_{z}$ on the GCS axis are related to the projections on the ACS axis $G_{N}, G_{L}, G_{E}$ by the following equality

$$
g=D(\alpha, \beta, \gamma) V^{T}\left(\Omega_{3} t, \varphi\right) G .
$$

The construction of a stochastic model of the NP it is acknowledged that due to internal and external disturbances accompanying the NP operation accelerations inevitably present in the astronomical coordinate system bear a random nature. With reference to the above equation (6) takes the following form:

$$
g=D(\alpha, \beta, \gamma) V^{T}\left(\Omega_{3} t, \varphi\right)(G+w),
$$

where $w$ is the vector of random disturbing accelerations, directed along the Earth trihedron axes. This vector is described by white Gaussian vector-noise (WGN) with zero mathematical expectation and known intensities matrix $D_{w}(t)$.

By combining equations (1), (2) and (7), the NP movement model may be represented as follows: 


$$
\begin{gathered}
\left(\begin{array}{c}
\dot{\alpha} \\
\dot{\beta} \\
\dot{\gamma}
\end{array}\right)=\Phi(\beta, \gamma)\left(R+U D(\alpha, \beta, \gamma) V^{T}\left(\Omega_{3} t, \varphi\right)(G+w)+\right. \\
\left.+\left(\left(D(\alpha, \beta, \gamma) V^{T}\left(\Omega_{3} t, \varphi\right)(G+w)\right)^{T} \hat{\otimes} K\right) D(\alpha, \beta, \gamma) V^{T}\left(\Omega_{3} t, \varphi\right)(G+w)+W\right),
\end{gathered}
$$

where $W-$ is the NP noise vector, also described as the WGN with zero mathematical expectations and the intensities matrix $D_{W}(t)$, or canonically,

$$
\dot{Y}=F(Y, t)+F_{0}(Y, t) \xi
$$

where $Y=(\alpha \beta \gamma)^{T}, \quad \xi=(w: W)^{T}$,

$$
\begin{gathered}
F(Y, t)=\Phi(\beta, \gamma)\left(R+U D(\alpha, \beta, \gamma) V^{T}\left(\Omega_{3} t, \varphi\right) G+\right. \\
+\left(\left(D(\alpha, \beta, \gamma) V^{T}\left(\Omega_{3} t, \varphi\right) G\right)^{T} \hat{\otimes} K\right) D(\alpha, \beta, \gamma) V^{T}\left(\Omega_{3} t, \varphi\right) G \\
F_{0}(Y, t)=\left(\Phi ( \beta , \gamma ) \left(U D(\alpha, \beta, \gamma) V^{T}\left(\Omega_{3} t, \varphi\right)+\right.\right. \\
\left.\left.+2\left(\left(D(\alpha, \beta, \gamma) V^{T}\left(\Omega_{3} t, \varphi\right) G\right)^{T} \hat{\otimes} K\right) D(\alpha, \beta, \gamma) V^{T}\left(\Omega_{3} t, \varphi\right)\right) \vdots \Phi(\beta, \gamma)\right)
\end{gathered}
$$

\section{Construction of Observation Equations}

In order to acquire a posteriori evaluations of the actual platform orientation we need a proper measuring instrument model that will take into account all the array of possible random impacts [2]. Further on the term measuring instrument will denote the complex of accelerometers positioned on a gyrostabilized platform. The relationship of acceleration projections along the platform trihedron axes may be established from Equation (6) by linearization of Matrix $D$. In this case Equation (6) takes the following form

$$
A=\tilde{D}(\alpha, \beta, \gamma) V^{T}\left(\Omega_{3} t, \varphi\right) G,
$$

where $\tilde{D}=\left(\begin{array}{ccc}1 & \gamma & -\beta \\ -\gamma & 1 & \alpha \\ \beta & -\alpha & 1\end{array}\right)$ - is Matrix $D(\alpha, \beta, \gamma)$, linearized with precision of the second

order infinitesimal angles. $A=\left(\begin{array}{lll}A_{x} & A_{y} & A_{z}\end{array}\right)^{T}-$ is the vector of accelerations measured by the accelerometers.

In order to simplify the further use of contemporary nonlinear stochastic filtration techniques in the resulting equation we will identify an addend, possessing no Vector $Y$ components. With that consideration Equation (10) takes the following form 


$$
\left(\begin{array}{l}
A_{x} \\
A_{y} \\
A_{z}
\end{array}\right)=\left(\begin{array}{l}
v_{1}^{*} \\
v_{2}^{*} \\
v_{3}^{*}
\end{array}\right)+\left(\begin{array}{ccc}
0 & -v_{3}^{*} & v_{2}^{*} \\
v_{3}^{*} & 0 & -v_{1}^{*} \\
-v_{2}^{*} & v_{1}^{*} & 0
\end{array}\right)\left(\begin{array}{l}
\alpha \\
\beta \\
\gamma
\end{array}\right),
$$

where

$$
\begin{gathered}
v_{1}^{*}=G_{N}\left(1-\cos \Omega_{3} t\right) \cos ^{2} \varphi+G_{N} \cos \Omega_{3} t+G_{L}\left(1-\cos \Omega_{3} t\right) \frac{\sin 2 \varphi}{2}+G_{E} \sin \varphi \sin \Omega_{3} t \\
v_{2}^{*}=G_{N}\left(1-\cos \Omega_{3} t\right) \frac{\sin 2 \varphi}{2}+G_{L}\left(1-\cos \Omega_{3} t\right) \sin ^{2} \varphi+G_{L} \cos \Omega_{3} t-G_{E} \cos \varphi \sin \Omega_{3} t \\
v_{3}^{*}=-G_{N} \sin \varphi \sin \Omega_{3} t+G_{L} \cos \varphi \sin \Omega_{3} t+G_{E} \cos \Omega_{3} t .
\end{gathered}
$$

Physical accelerometers inevitably feature their own random measurement noise generated by their random operational environment impacts such as heat, electrical magnetism, gravity, etc. [2]. In this case the model of the signal measured by the accelerometer triad may be represented by the following equation

$$
\left(\begin{array}{l}
Z_{A_{X}} \\
Z_{A_{y}} \\
Z_{A_{z}}
\end{array}\right)=\left(\begin{array}{l}
v_{1}^{*} \\
v_{2}^{*} \\
v_{3}^{*}
\end{array}\right)+\left(\begin{array}{ccc}
0 & -v_{3}^{*} & v_{2}^{*} \\
v_{3}^{*} & 0 & -v_{1}^{*} \\
-v_{2}^{*} & v_{1}^{*} & 0
\end{array}\right)\left(\begin{array}{l}
\alpha \\
\beta \\
\gamma
\end{array}\right)+\left(\begin{array}{l}
W_{A_{x}} \\
W_{A_{y}} \\
W_{A_{z}}
\end{array}\right)
$$

where $Z_{A_{i}}, i=x, y, z-$ are the signals measured by $i$ - accelerometer, $W_{A_{i}}-$ own measurement noises of the $i$ - accelerometer, approximated by the WGN with zero mathematical expectations and the intensities matrix $D_{W_{A}}(t)$.

In the vector-matrix form Equation (12) may look as follows

$$
\tilde{Z}_{A}=M_{0}+H(t) Y+W_{A}
$$

where $M_{0}=\left(\begin{array}{c}v_{1}^{*} \\ v_{2}^{*} \\ v_{3}^{*}\end{array}\right), H(t)=\left(\begin{array}{ccc}0 & -v_{3}^{*} & v_{2}^{*} \\ v_{3}^{*} & 0 & -v_{1}^{*} \\ -v_{2}^{*} & v_{1}^{*} & 0\end{array}\right)$.

Or, canonically

$$
Z_{A}=H(t) Y+W_{A}
$$

where $Z_{A}=\tilde{Z}_{A}-M_{0}$.

Equations (9) and (14) represent an NP movement model in the object-observer form. The use thereof allows for evaluating State vector (9) with the help of modern nonlinear stochastic evaluation techniques [4]. The a posteriori probability density $\rho(Y, t)=\rho$ of this process has the following form [4]:

$$
\frac{\partial \rho}{\partial t}=-\sum_{i=1}^{3} \frac{\partial}{\partial Y_{i}}\left[q_{i}(Y, t) \rho\right]+\frac{1}{2} \sum_{i=1}^{3} \sum_{j=1}^{3} \frac{\partial^{2}}{\partial Y_{i} \partial Y_{j}}\left[b_{i j}(Y, t) \rho\right]+[F(t, Y)-F(t)] \rho(Y, t),
$$


where

$$
\begin{gathered}
q_{i}(Y, t)=F_{i}(Y, t)+\frac{1}{2} \sum_{k=1}^{3} \sum_{j=1}^{3} D_{k} F_{0 j k}(Y, t) \frac{\partial}{\partial Y_{j}} F_{0 i k}(Y, t), \\
b_{i j}(Y, t)=\sum_{k=1}^{3} D_{k} F_{0 i k}(Y, t) F_{0 j k}(Y, t), \\
F(Y, t)=-\frac{1}{2}[Z-H(Y, t)]^{T} D_{\zeta}^{-1}[Z-H(Y, t)], F(t)=\int_{-\infty}^{+\infty} \int_{-\infty}^{+\infty} \int_{-\infty}^{+\infty} F(Y, t) \mathrm{\rho} d Y_{1} \ldots d Y_{R} .
\end{gathered}
$$

And now we will establish an a posteriori moments system that will equip us with the required approximation precision for Equation (15) in the given time interval of the system operation (9). In order to solve the set task we will employ the technique outlined in [4,5]. We will proceed by multiplying the both sides of Equation (15) by $Y_{k}, k \in \overline{1.3}$ and integrating the result by $Y_{1} Y_{2} Y_{3}$. With consideration that changing the integration and differentiation order in the left side of the equality is admissible we achieve the following:

$$
\int_{-\infty}^{+\infty} \int_{-\infty}^{+\infty} \int_{-\infty}^{+\infty} \frac{\partial \rho}{\partial t} Y_{k} d Y_{1} d Y_{2} d Y_{3}=\frac{\partial}{\partial t} \int_{-\infty}^{+\infty} \int_{-\infty}^{+\infty} \int_{-\infty}^{+\infty} \rho Y_{k} d Y_{1} d Y_{2} d Y_{3}=\frac{\partial}{\partial t} m^{k}=\dot{m}^{k} .
$$

Using the technique of integration by parts, considering the borderline conditions and drift and diffusion coefficients Taylor series expansion, the first addend (drift coefficient) in the right hand side of Equality (15) will be represented as $\sum_{n=0}^{\infty} a^{k} m_{n}^{k}$, where $a_{n}^{k}=\frac{\partial^{n} q_{k}(m)}{\partial Y_{k}^{n}} \frac{1}{n !}$ . According to the above considerations the second addend in (15) (diffusion coefficient) turns to zero, and the third addend in (15) may be written as $\sum_{n=0}^{\infty} f_{n}^{* k} m_{n}^{k}$, where $f_{n}^{* k}=\frac{\partial^{n}\left([F(Y, t)-F(t)] Y_{k}\right)(m)}{\partial Y_{k}^{n}} \frac{1}{n !}$. The combination of the resulting expressions helps us acquire the equations for the initial moments of Phase coordinates $Y_{k}$

$$
\dot{m}^{k}=\sum_{n=0}^{\infty} a_{n}^{k} m_{n}^{k}+\sum_{n=0}^{\infty} f_{n}^{* k} m_{n}^{k}
$$

In order to find the central moments Equality (15) will be multiplied by $\left(Y_{k}-m^{k}\right)^{j}$ and subsequently integrated by $Y_{1} Y_{2} Y_{3}$. The above transformations will result in the following

$$
\dot{m}_{j}^{k}=j \sum_{n=0}^{\infty} a_{n}^{k} m_{n+j-1}^{k}+\frac{j(j-1)}{2} \sum_{n=0}^{\infty} b_{n}^{k} m_{n+j-2}^{k}+\sum_{n=0}^{\infty} f_{n}^{k} m_{n}^{k}, j>1
$$


It should be noted that in this case the diffusion coefficient is rearranged into $\frac{j(j-1)}{2} \sum_{n=0}^{\infty} b_{n}^{k} m_{n+j-2}^{k}$, where $b_{n}^{k}=\frac{\partial^{n} b_{k k}(m)}{\partial Y_{k}^{n}}$, and the Taylor series expansion coefficients for the latter addend are determined as follows: $f_{n}^{k}=\frac{\partial^{n}(F(Y, t)-F(t))(m)}{\partial Y_{k}^{n}} \frac{1}{n !}$.

In order to find the product moments (15) is multiplied by $\left(Y_{i_{0}}-m^{i_{0}}\right)^{j}\left(Y_{j_{0}}-m^{j_{0}}\right)^{s}$, and the result is integrated by $Y_{1} Y_{2} Y_{3}$ to achieve the following

$$
\begin{aligned}
& \dot{m}_{j, s}^{i_{0}, j_{0}}=-j \sum_{n=0}^{\infty} a_{n}^{i_{0}} m_{n+j-1, n}^{i_{0}, j_{0}}-s \sum_{n=0}^{\infty} a_{n}^{j_{0}} m_{j, n+s-1}^{i_{0}, j_{0}}+\frac{1}{2}\left(j(j-1) \sum_{n=0}^{\infty} b_{n}^{i_{0}} m_{n+j-2, s}^{i_{0}, j_{0}}+\right. \\
& \left.+s(s-1) \sum_{n=0}^{\infty} b_{n}^{j_{0}} m_{j, n+s-1}^{i_{0}, j_{0}}+j s \sum_{n=0}^{\infty} b_{n}^{i_{0} j_{0}\left(j_{0}\right)} m_{j-1, n+s-1}^{i_{0}, j_{0}}+j s \sum_{n=0}^{\infty} b_{n}^{j_{0} i_{0}\left(i_{0}\right)} m_{n+j-1, s-1}^{i_{0}, j_{0}}\right)+\sum_{n=0}^{\infty} f_{n}^{i_{0}} m_{j+n, s}^{i_{0}, j_{0}} \\
& \text { where } a_{n}^{i_{0}}:=\frac{\partial^{n} q_{i_{0}}(m)}{\partial Y_{i_{0}}^{n}} \frac{1}{n !}, a_{n}^{j_{0}}:=\frac{\partial^{n} q_{j_{0}}(m)}{\partial Y_{j_{0}}^{n}} \frac{1}{n !}, b_{n}^{i_{0}}:=\frac{\partial^{n} b_{i_{0} i_{0}}(m)}{\partial Y_{i_{0}}^{n}} \frac{1}{n !}, \\
& b_{n}^{i_{0} j_{0}\left(j_{0}\right)}=\frac{\partial^{n} b_{i_{0} j_{0}}(m)}{\partial Y_{j_{0}}^{n}} \frac{1}{n !}, i_{0}, j_{0} \in \overline{1,3}, s>1 .
\end{aligned}
$$

The combination of Equations (17), (18) and (19) results in a nonlinear filtration algorithm free of the inherent drawbacks of the Kalman filtering algorithms and requiring no a priori assumptions on the Gaussian distribution of the a priori probability density of a dynamic stochastic system:

$$
\begin{gathered}
\dot{m}^{k}=\sum_{n=0}^{\infty} a_{n}^{k} m_{n}^{k}+\sum_{n=0}^{\infty} f_{n}^{* k} m_{n}^{k} \\
\dot{m}_{j}^{k}=j \sum_{n=0}^{\infty} a_{n}^{k} m_{n+j-1}^{k}+\frac{j(j-1)}{2} \sum_{n=0}^{\infty} b_{n}^{k} m_{n+j-2}^{k}+\sum_{n=0}^{\infty} f_{n}^{k} m_{n}^{k} \\
\dot{m}_{j, s}^{i_{0}, j_{0}}=-j \sum_{n=0}^{\infty} a_{n}^{i_{0}} m_{n+j-1, s}^{i_{0}, j_{0}}-s \sum_{n=0}^{\infty} a_{n}^{j_{0}} m_{j, n+s-1}^{i_{0}, j_{0}}+\frac{1}{2}\left(j(j-1) \sum_{n=0}^{\infty} b_{n}^{i_{0}} m_{n+j-2, s}^{i_{0}, j_{0}}+\right. \\
\left.+s(s-1) \sum_{n=0}^{\infty} b_{n}^{j_{0}} m_{j, n+s-1}^{i_{0}, j_{0}}+j s \sum_{n=0}^{\infty} b_{n}^{i_{0} j_{0}\left(j_{0}\right)} m_{j-1, n+s-1}^{i_{0}, j_{0}}+j s \sum_{n=0}^{\infty} b_{n}^{j_{0} i_{0}\left(i_{0}\right)} m_{n+j-1, s-1}^{i_{0}, j_{0}}\right)+\sum_{n=0}^{\infty} f_{n}^{i_{0}} m_{j+n, s}^{i_{0}, j_{0}}
\end{gathered}
$$

\section{Conclusion}

The system of a posteriori moments (20) is potentially capable to provide a precise solution of Equation (15) for the given time interval of a stochastic dynamic system (9) operation and allows to take into account the existing correlations in its state vector variables. For cases when the a posteriori probability density of a process may be adequately approximated by 
normal distribution density depending on the first two moments only, the resulting equation system reduces to Kalman-type filtering.

\section{Acknowledgements}

This research was supported by RFBR Grant № 19-01-00451.

\section{References}

1. Sokolov S V, Pogorelov V A 2016 Stochastic Evaluation, Control and Identification in High-precision Navigation Systems (Moscow: Publ Fizmatlit) p 264

2. Sokolov S V, Pogorelov V A 2009 Fundamentals of Multi-Structural Strapdown Navigation Systems Synthesis (Moscow: Publ Fizmatlit) p 190

3. Rozenberg I N, Sokolov S V, Umanskij V I, Pogorelov V A 2018 Theoretical Basis of Close Integration of Inertial Satellite Navigation Systems (Moscow: Publ: Fizmatlit) p 312

4. Sinicyn I, Kalmann N and Pugachev N 2006 Filters (Moscow: Publ Logos) p 640

5. Mit'kin A S, Pogorelov V A, Chub E G 2015 Radiophys Quantum El 58 224-232 